Journal of

Cancer Research and Therapeutic Oncology

\title{
Cancer as an Emergent Phenomenon?
}

\author{
Anderson $\mathrm{KM}^{1, *}$, Patel $\mathrm{MK}^{2}$ \\ ${ }^{1}$ Rush Medical College (Emeritus) the College of Nursing \\ ${ }^{2}$ University of Illinois, both in ChicagoI 160612
}

${ }^{\star}$ Corresponding author: Anderson KM, Rush Medical College (Emeritus)the College of Nursing; E-mail:kanderso427@sbcglobal.net

Received Date: September 14, 2018; Accepted Date: October 30, 2018; Published Date: November 01, 2018

Citation: Anderson KM (2018) Cancer as an Emergent Phenomenon?. J Cancer Res Therap Oncol 6: 1-9.

\begin{abstract}
Many biological events exhibit major features of emergent phenomena in which the detailed behavior of a collective ensemble of entities or agents is not predictable from summing their individual behaviors. The relationship between the emergent phenomena of evolution, including the development of cancer cells expressing apparently "random" genetic / epigenetic events and the influence of "scale" are also not yet widely understood or well defined. Presumably this uncertainty is also subject to further influence from non-oncologic random genetic and epigenetic events. A forward or a retrogressive evolution of individual cancer cells could be viewed as subsets of emergent phenomena in which random genetic / epigenetic events can also meld with other ongoing, underlying emergent processes, contributing to unpredictable outcomes. The extent to which such an interplay may subvert attempts at cancer therapy is not presently well identified nor are conditions that might promote or retard the incidence of random responses during the process. The rare reports of what may have included occasional random reversals of established cancers is another subject of interest. Some discussion of these questions seems merited.
\end{abstract}

Keywords: Emergence, Evolution, Cancer

\section{Introduction}

\section{Emergent Phenomena}

Several properties characterize emergent phenomena [1-6]. "Strong" emergent phenomena originate from collective systems in which the resulting form and behavior of its aggregate could not have been reliably predicted from the individual properties of its precursor elements. "Weak" emergent phenomena exhibit properties that can be at least suggestive of those exhibited by their precursor elements $[1,3,4]$. For example, individual cardiac myocytes exhibit spontaneous contractions which can be synchronized. In bulk and properly organized with a vascular system, they compose a propulsive organ, the heart, able to propel blood through a vascular system. In this case, while the aposteriori result of the collective outcome reflects a behavior of its individual members suggestive of their individual capability, details of the form it finally takes, number of cardiac valves, chambers, conduction system, etc. vary among the life forms employing

(C)2018 The Authors. Published by the JScholar under the terms of the Creative Commons Attribution License http://creativecommons.org/licenses/by/3.0/, which permits unrestricted use, provided the original author and source are credited. variants of such propulsive systems, and would not generally have been predictable.

"Strong" emergent phenomena can include radical novelty, dynamism with an underlying quasi-stability, an imbalance with the whole exceeding the qualities and apparent capacities of any sum of its parts. Some outcomes may reflect a retrogressive "blocked" development $[7,8 \mathrm{a}, \mathrm{b}]$ and others that might be termed a "forward" causation with the acquisition by tissue stem cells [9-11] of new, at times radical modifications of metabolic pathways able to activate nascent or evoke new, contingent outcomes [12].But the developing behavior of large, emergent ensembles capable of radical emergent behavior generally is not evident from any obvious predictable sum or difference of properties exhibited by their individual constituents. What has seemed even more puzzling, such considerations apply to both animate and inanimate systems and can influence many very large collections of similar components inherently capable of dynamic interactions [1, $3,4]$. It appears that many large ensembles of animate or in animate entities exhibiting certain Inherent capacities are subject to a similar form of a universal, endogenous "logic", "program", "protocol" or "code" when confronted by certain 
unidentified endogenous capacities of the interacting entities. And these outcomes may become evident over prolonged intervals of time or in other situations within several generations, e.g. Galopagos pigeon beak morphology. Such events seem to represent fairly "random" happenings, which however are consistent with and can promote (or retard) augmented survival.

It may not be surprising that the behavior of large "ensembles" of "entities" usually cannot be characterized by summing individual properties of their constituents due to the complexity of their random interactions. The interactions are too numerous and unique interactions can occur as rare events. These constituents include atoms, molecules, components of the weather, the stock market, behavior by collections of birds, fish, and many animate and inanimate, biological or physical systems An example such as the behavior of the stock market is instructive. While it exhibits characteristics of emerging phenomena, it is also subject to behaviors resembling those of a statistical random walk, suggesting a merging of differing strains of influence on its overall behavior. It appears that if a large and rich enough collection of interactions between constituent components is capable of occurring, the complexity of the multitude of interactions preclude reliable predictions of group behavior, due to an impossible number of unknown interactions to try and identify, calculate and model the system under study.

While evolutionary pathways underlying the myriad of prior and extant life- forms qualify as a continuing series of inter-related emergent events, we suppose that the origin and progression of malignant disease generally represents a subset of disordered events in these prior and contemporary, larger inter- related programs of ongoing organic evolution, expressed by cells exhibiting a mis-programmed reproductive advantage associated with an increased survival of the "fittest". Such changes can occur over a number of years $(13,14$ a - c, $32 \mathrm{~b}$ ); others in a lesser time span, as in childhood or even in utero.

\section{Explanations of Emergence Including The Law Of Large Numbers, Complexity Theory And Scale As Applied To Macroscopic and Microscopic Biological And Other Events}

The study of complexity provides insight into the behavior of very diverse systems that can seemingly be dictated by elements of a common logic involving large numbers of interacting elements $[15,16 a, b, 19]$. The "logic" of cancer presumably encompasses defects at cellular genomic and extra-genomic signal transduction, structural assemblies and their function, etc. as they may alter the function of diverse intra and inter cellular networks, sub-networks, modules and nodal interactions related to cellular replication and differentiation $[16 \mathrm{~b}, 17]$. The influence of scale relies upon the law of allometry, that in many growing systems, some of the elements associated with growth are non-linear [16a]. In addition to unidentified combinatorial events, cellular history can also be affected by additional broadly defined random events, including "mutations" and other biochemical interactions involving various sorts of genetic and epigenetic mechanistic "errors" and instability $[6,11,17,28,30]$.

The "law of large numbers", essentially the addition of small Gaussian uncertainties, really a statement of the central limit theorem of statistics, can be invoked to explain an effect of aberrant cells on a population's subsequent composition $[18,19]$. Individual variations are summed in bulk, in a kind of mass-action averaging, the outcome however appearing as deterministic due to the large number of cells expressing behaviors close to some broad average. To employ an earlier analogy [20], individual molecules of water in a wave are distinctive in many ways; positions, momentum, number of interactions with other water molecules, etc., perhaps even somewhat chaotic, yet the wave eventually reaches the shore, a deterministic outcome. It does so due to an overall structure related to relationships between the much larger numbers of more average molecules of water, a structure sufficient to dilute out effects, as it were, of the lesser number of outliers, unless their effect or numbers were sufficiently disruptive to disturb the majority forces maintaining the behaviors of the much more numerous molecules tending toward the "average". This amounts to a representation of the central limit theorem of statistics.

Attempts to understand the origin of emergent phenomenon have included elements of statistical and physical explanations, statistical mechanics, elements of the complexity theory and even quantum theory [14-17].

\section{Bifurcation Diagrams}

If a "choice" for a system is to take one of several future options, a "fork" in the road, so to speak, beyond that point the properties of the system can change quickly. In a Feigenbaum bifurcating cascade representing an unfolding of a hypothetical cellular history, a non-linear system undergoes multiple periodic doublings, which can eventuate in a chaotic response [21]. These representations provide a means of visualizing multiple potential responses by members of an "ensemble". Period doubling bifurcations represent different collections of states becoming available to members of the system. "Attractors" are employed to describe behavior of such dissipative systems: fixed point attractors correspond to equilibrium and steady states, limit cycle attractors to periodic states and strange attractors to chaotic states which are especially sensitive to initial conditions [21]. In addition, limit cycle attractors exhibit Mandelbaum fractal properties, repetitive self- similarity at all scales of observation [21,22, 23 $\mathrm{a}, \mathrm{b}]$.

Chaos is characterized by apparently random, unpredictable behavior and includes categories of dissipative chaos and deterministic chaos [21-25] Dissipative structures represent organized matter beyond the first bifurcation not at thermodynamic equilibrium. Deterministic chaos contains some predictable elements such as deterministic non-linear dynamical equations changing over time This is distinguished from random or "stochastic" changes in the environment, 
so called "stochastic chaos", equated to "noise" that includes seemingly random behavior but is unrelated to a strange attractor [26, 27]. In conservative (classical) dynamic systems, time is reversible, equations work in both directions. In dissipative systems, time is irreversible. A dissipative system, a state of matter arising after the first bifurcation, is maintained far from thermodynamic equilibrium [21]. According to the "irreversibility paradox", "macroscopic" systems are timeirreversible, e.g., we all age and die, while "microscopic" systems are time-reversible. In addition, both statistical mechanics and chaos are considered deterministic but not predictable; quantum mechanics seems to be considered neither deterministic nor predictable. We leave the paradox unresolved [29c, d].

These definitions, extracted from the references indicated [3, 15, 16, 25-27] several from Wikipedia, authors unknown, provide some indication for the direction of further study. Any confusion or mis-understandings off these terms likely is due to our attempts to paraphrase them.

\section{Summary}

Up to this point the intention has been to relate some of these ideas to the logic underlying the origin and progression of cancer as a byproduct of evolutionary events. Combined with concepts involving gene networks, nodes, computer programming and related informational and mathematical concepts it has not been farfetched to envision genetic / biochemical circuitry with a resemblance to electronic "circuitry", feedback, hysteresis, aspects of computer programming and the details of complexity and network theory as underlying a descriptive "logic" of cancer biology[15-28].

It might seem odd that random genomic and epigenomic events can lead to populations of cells exhibiting deterministic outcomes affecting their survival. If affected cells initially are few in number, stochastic effects can be disproportionately influential. While the final population resulting from a small population of original cells can be uncertain due to genetic drift, a random change in the frequency of a robust gene-variant in a small population could outgrow their un-affected companions, eventually becoming the dominant clonal representative.

By way of a further summary: Large ensembles can represent much more than an apparent sum of their constituents, depending upon the past, present or future combinatorial possibilities within the new daughter cells and vis a' vis utilization of much of the genetic and functional details acquired from the initial malignant parental stem or other cell of origin, all occurring against the backdrop of random mutational and epigenetic errors occurring in both sets ofany involved parental cells and their subsequent progeny. Under those conditions an extended exposure to chance oncogenic modifications of them and their progeny's inheritances can occur over evolutionary time.

\section{Origins of Malignant Disease}

What follows are a few very introductory generalities regarding some major features of malignant disease. Currently, cancer causation is ascribed to hereditary $(\mathrm{H})$, environmentally induced (E) or randomly -induced ( $\mathrm{R}$ ) forms $[6,12,18,28]$, The likely contribution of each category in a given instance is arguable, with many favoring environmental causes overall. If responsible environmental agents are identified, associated cancers should be avoidable, perhaps an overly-optimistic point of view due to many reasons. Others believe that many cancers originate from random errors in DNA synthesis or repair [28]. A correlation has been demonstrated between the estimated number of cellular replications occurring over the lifetime of a tissue and the likelihood of a cancer originating from that source [29a,b] although this is not universally agreed with. Random (or "stochastic") events affecting other cellular components include epigenetic events that also can be causative (30).An additional uncertainty is whether the precancerous "target"- cell(s) usually represents a cell or tissue stem cell or a partially differentiated somatic cell; hierarchically- oriented pathways of very ancient origin, "from the top" or a retrogression or "blocked" differentiation of a partially differentiating somatic cell either experiencing defective replication or defective differentiation [31 a $\mathrm{d}, 32 \mathrm{a}, \mathrm{b}]$. Whatever the details, malignant changes occur against a backdrop of such underlying events.

\section{Some Implications Inferred From the Preceding: Different Roles for Chance In "Normal" Or "Oncogenic" Emergence}

It is believed that a dividing stem or developing somatic cell will expresses a complicated series of events, pre-programmed by eons of prior hereditary history of its predecessors. In principle these events could be describe-able by a complicated "program" involving the interplay of genetic and epigenetic assemblies and sub-assemblies, modules, networks of interrelated nodes and intra and inter-cellular subsystems responsible for implementation of necessary tasks $[16 \mathrm{a}, 33]$. Since there is general agreement that mutational and related events are responsible for cellular evolution and for the initiation and maintenance of malignant changes $[6,12$, 18 ], these must occur before, during or possibly even after the "emergence" of replication and development. Altered events during non-malignant "emergence", occurring in parental or progeny cells, should differ qualitatively from "oncogenic" mutational and associated events in either population. Nonmalignant emergence would seem to be accompanied by more limited developmental changes, that is, cell lines appear to breed phenotypically "true"; "oncogenic" developmental events should be more radical and extreme. Whether they can stand "alone" or require some prior form of complementary changes in genomic potential derived from the parental line(s) may distinguish several forms of oncogenic histories. The interaction between daughter cells and the genetic potential of precursor parental cell(s) would seem something of a two- way street. Newly developed genomic or other changes would need to be able to interact with major elements of the 
residual developmental programming in the parenteral stem or involved somatic cells.

One way to categorize random genomic and epigenetic changes is according to a hierarchical scheme [20, $32 \mathrm{a}, \mathrm{b},, 33$, $34 \mathrm{a}, \mathrm{b}]$. Random Housekeeping events (type 1) affect basic survival requirements of differentiated cells; DNA replication and repair, basic energy provision and its modulation, factors affecting influx and efflux of critical metabolites, etc. Interaction partners could express altered activity of enzymes, signal transduction, membrane components and the like but no long term changes in cell structure or function might be implied. Random Tactical Events (type 2 events) would include more complex changes affecting signal transduction, DNA synthesis, repair, or other diverse responses to more serious cellular stresses, that may involve cellular, network, node and other components of a cellular program required for replication and survival. Changes at the network, inter or intra- network and sub-networks could reflect more permanent interactions. These changes might involve components involved in the cellular programming responsible for replication, repair and survival. Finally, Random Strategic changes (type 3) would include fundamental modifications including initiation of new pathways superimposed on more ancient developmental circuitry. Over time, gene or chromosome duplications or deletions could mandate major re-direction of cellular evolution. Recently, "supergenes", a group of genes in chickens were described that are transferred as if one or more genes are inverted in a "flipped over" form [34]. This inversion, at least initially prevents these genes from combining with those of its matching chromosome, nor are they as susceptible to repair mechanisms but are presented as a "package" to daughter cells. As such genes evolve divergently they are thought able to contribute to unexpected outcomes, modifying the subsequent development of daughter cells in unique ways.

It is tempting to consider that the third proposed category might actually be followed by a fourth category of Chaotic Randomness (Type 4). Such a category mandating more extreme disruption of genomic organization may be required for radical reorientations of evolutionary direction involving such profound events as creation of new species, the formation of new genera and the like [35 a, b, 50]. Development of bilateral symmetry, dorsal- ventral or antero-posterior differences or other major forms of structural and functional reorganization and development of new forms of biological entities could be included. The capacity to undertake radical rearrangements of genomic material may be just what was required to create and sustain events such as the punctuated equilibria of current evolutionary theory [ $8 \mathrm{a}, \mathrm{b}, 49]$. It is less clear that ongoing development of most cancers would normally require such radical developments to acutely evolve over the lifetime of a hypothetical patient.

Regarding the frequency of regulatory or other random events, a proposed ordering of $1>$ or $=2>3$ >>>> 4 would seem feasible. These categories might "blend" somewhat. Examples of several events in the same category or in a mixing between events in several categories might occurred in suitably affected cells. A cancer cell is a form of parasite, and with the death of its host, that resident clone dies out unless its' potential was previously passed on to progeny. Examples of intra-species transfer of cancers are rare, e.g., the Tasmanian facial tumor [36] or possibly occasionally via organ transplants. Uptake of DNA released by contiguous cells in a tissue has been also been described.

A mutational event might occur without immediate consequence. Some future additional event able to interact with the prior one, either activating or inhibiting some third event could have important consequences for the further "emergence" of the affected cell.

As mentioned, it has also been speculated whether the biological equivalence of a limited form of regulatory chaos is the ultimate behavior underlying the suggested third and even more extreme fourth more radical form of random, strategic evolutionary change fundamental to major developmental changes in cellular structure and function, leading to the extensive variability and range of evolutionary outcomes [ $8 \mathrm{a}, 35 \mathrm{a}, \mathrm{b}, 49]$. The occurrence of enormous numbers of interacting cellular regulatory and metabolic components in new combinations with unique admixtures of contingent events generates very large numbers of potential permutations and combinatorial interactions over time. While many details of such interactions in cells thought to be undergoing an emergent development are obscure, something like this may lie at the core of similar outcomes in cellular differentiation $[10,11,12 \mathrm{a}, \mathrm{b}, 35,37,38]$, formation of new biological species and genera and in other disparate fields in which emergent outcomes also occur.

\section{Necessary Integration of Oncogenic and Non- Oncogenic Random Genomic / Cellular Changes}

Whatever the random changes in either parents or progeny, the resulting clones will in some manner have to be integrate-able with the extant evolutionary history of its' parental line. Unless the modified cell line genome survives in progeny and is able to undergo extended evolutionary changes, evidence of random changes can be lost in the short term, especially in older post-reproductive cancer patients. Changes might occur in non-oncogenic regions of the cell, yet leaving it and its progeny sufficiently similar phenotypically to the parental line. Acquisition of unusual growth properties could signal a capacity for malignant behavior. Changes that are too radical might experience long delays or even eventual failure in attempts at re-integration with components of the original parental origin. Radically new features, such as a propensity to be less responsive to Internal or external influences normally limiting their replication, extent of differentiation, modification of old or introduction of new signal transduction or other pathways and their implementation and for more aggressive cells with an ability to metastasize would seem to distinguish a limited number of developing aggressive cells from less aggressive progeny. The development of such "radical" events would be consistent with a developing current or future "strong" emergence. 
Global properties of an evolving ensemble are often distinct from those of its individual components. The theories underlying complexity provides part of an understanding

The appearance of emergence in both diverse animate and inanimate systems seems it's most remarkable feature. The "entities", include atoms, molecules and various other animate and inanimate entities, and explanations for the responses when large collections (ensembles) are studied has presented a persistent puzzle $[1-4,16, b, c]$. Large numbers of "entities" seemingly follow their own forms of a universal logic, alluded to previously. Examples of and partial explanations for emergent phenomena are diverse, associated with unexpected results in the stock market, weather forecasting, the behavior of many purely physical phenomena and many animal behaviors, e.g. flocks of birds, schools of fish, etc. [1 - 4,15,16a - c,19,22,25]. Questions as to why and how this occurs remain arguable.

It should also be mentioned that examples of similar cancers can arise from different cellular origins, sharing similar molecular details. Thus recently, human cultured epithelial prostate and lung cells have been altered into cells expressing a neuroendocrine small cell cancer lineage, following expression of a group of common "driver" factors [51 a ,b]. Whether transformed cells respond to subsequent responses from stochastic events, as distinct from any that may have contributed to the original induction of malignant transformation could be of interest.

\section{Cancers as emergent phenomena?}

To return to the original question; as benign or malignant cancers evolve, do they develop properties of strong or weak emergence? When compared with organic evolution, it seems inevitable to view the underlying play of events in oncology to have included apparently random changes in past, current or future normal development. There seems to be general agreement that "chance", randomness, stochasticity and presumably even chaos have contributed to and are even responsible for the enormous variety and uniqueness in the multitude of different life forms.

To review: diverse inanimate and animate populations composed of individual "units" provide opportunities for very large numbers of interactions among and within their members, leading to unanticipated outcomes that often do not directly mirror properties of the individual units. It may be the case that developmental programs participating in "housekeeping" and random level 2 random "tactical" changes, would be more compatible with "weak" emergence, possibly also including clinically "benign tumors" exhibiting more restrained growth. More radical, fundamentally level 3 random "strategic" modifications or even an influence of chaotic events able to successfully reintegrate with the parental genome would more likely be associated with a "strong" emergence. A comparison at genomic and extra-genomic expression in cells exhibiting ostensibly "weak" emergence with purported "strongly" emerging cells could clarify the nature and the extent of these behaviors.
It can be remembered that cancers arising de novofrom somatic cells without an association with procreative cells would seem only capable of a more limited form of evolutionary development unless the cancer information could be passed on to progeny. Reproductive cells or their progeny containing potential oncogenic information should have a much greater potential to undergo serial emergent developmental events, including stochastic changes relevant for oncogenesis; cancer viewed as an ongoing developmental inborn error in these cells.

From our ethnocentric point of view, the fortunate inability of mammalian cancers or their DNA to be transferred to, or as it were to "infect" other members of homosapiens [31 c], fundamentally delimits the ability of a clinically established cancer to undergo aprotracted, interactive development of organic evolution occurring over evolutionary time. While stochastic events no doubt occur during their lifetimes, their accumulated number is limited due to the death of the host and lack of reproductive continuity for genomes of the affected cancer cell in pre or post-fertile patients. A truly parasitic existence of cancer cells could have briefly extended their survival in an individual patient.

The genesis of any ability to bypass immune rejection by a potential host would have represented a catastrophic development in human history, and might eventually have ended it. Transmission of human cancers to fellow humans or other animal cancers to the same or other species does not seem to have occurred except in a few instances, e.g., facial cancer in Tasmanian devils and canine transmissible venereal disease [36].

While cancers believed to originate from a common stem or a somatic cell share many common properties; genomic, and presumably epi-genomic "signatures" in different regions of the cancer can differ significantly [13,14a-c,15]. In addition, the signatures from metastases can also differ, both from the primary deposit and from one another $[13,14 \mathrm{a}-\mathrm{c}]$. The ability to compare genomic signatures in single cells, employing single cell RNA-seq technique coupled with identification of unique, single clone-dependent differences with the parental line of cells and clustering algorithms to identify individual cell types combined with DNA sequence information should provide more definitive evidence of the presence of stochastically dependent malignant evolution and its mechanisms consistent with the properties of emergence [40 a, b,43]. Such evidence does not seem to be available, or at least is unknown to us. No two individuals, especially those derived from a monozygote, are truly "identical' at all molecular levels [44 a-c, 45]. The widespread evidence for mosaicism, the presence of cells with differing genomic heritages in an organism [46 a, b], is an additional argument against the supposed informational identity of what may appear to be "identical" cells. Despite the many superficial phenotypic similarities of identical mono-zygotically-derived twins, rendering a macroscopic comparison almost convincing, evidence of dis- similarity at microscopic genomic levels seems to obtain [ $44 \mathrm{a}, \mathrm{c}, 45$ ]. 
To the extent that deviant development of cancer progeny is subject to random genomic or epi-genomic variation, evolution of sub-species of cancers believed to originate from a given malignantly transformed stem or somatic cell can be anticipated. Are any examples of "selfcorrecting" mutations known? Rare examples of what were believed to be "spontaneous" regressions (including "cures" ?) of cancer might have represented examples of this phenomena [47], but we are unaware of a published example in which a specific example or mechanism has been demonstrated which "spontaneously" achieved this result. Sickle cell anemia in which one disease process provides limited protection against another disorder, malaria, represents a hint of mechanisms that may become available to limit or even reverse an underlying malignant process [48]. Therapeutic attempts to reverse or otherwise interfere with a specific identified malignant "signature", e.g. targeted chemo-therapeutic agents, modification of gene readouts, etc., mimic such an approach.

Ideally, the ability to closely identify unique genomic/ functional differences employing single cell RNA sequencing studies and DNA sequence information may identify differences in malignant behavior and response to therapy reliant on stochastic events between individual progeny from malignantly transformed cells that distinguish them from their precursors. Were radical events such as essential gene or chromosomal duplications or deletions to occur, the expectation is that the two populations, given time would become more widely divergent. However any influence on future organic evolution should be nil and of an indirect nature unless the changes can be retained and contribute to future cellular evolution

If the core explanation underlying emergence is the development of a large population of malignantly transformed or of normal cells (or other entities, weather events, the stock market etc.),with numerous interacting components subject to an enormous multiplicity of random interactions super-imposed upon existent developmental programs, with unpredictable outcomes, some sense of why and how large collections of "participant entities" subject to unexpected combinations becomes intellectually more tractable. However simplistic, this argument provides some sense of how unique outcomes could become imposed upon resident developmental programs. The nature and frequency of these contingent interactions remains largely unknown and of course, unpredictable.

Some form of a " universal" logic [16a],seemingly unlike that we usually associate with "macroscopic" physical events seems to exist between the macroscopic and the "microscopic" scales and when very large numbers of participating entities express an ability to interact in unexpected ways, generating totally new combinations of biologic and non-biologic realities [16 a - c, 23-25 a, b]. The nature of these interactions and their effects on subsequent developmental events presents further features to be understood. Possibly distinction between noise associated with biological events [26,27], chaos versus randomness [ 25], quantum Bayesian logic [25], deterministic chaos [16], dissipative chaos [2], stochastic optimization [8b], chaos versus randomness $[5,24,25]$ or additional differentiations of chance and relativistic chaos [21] will provide further insight. It seems that mathematicians generally equate randomness and stochasticity while physicists often make further distinctions [25 b]. In distinguishing random from chaotic data, a deterministic system should have a small, stable error, or if increasing exponentially exhibit chaotic behavior; a stochastic system would exhibit a randomly distributed error [25 a, b]. Random processes are irregular and unpredictable, chaotic ones are irregular, deterministic, impossible so far to predict, complex but ordered and may be associated with a fractal structure [16b, c, 25, 35]. It seems curious that stochasticity could contribute to seemingly non-random and even to chaotic biological outcomes associated positively with a temporary deterministic survival of the "fittest" cancer cells, although this result is so negative for a patient [4]. However, if even this ability had not evolved, there might not have been any human history [50]. That is, stochasticity can "cut" both ways.

\section{CODA}

Commenting on subjects in which one has superficial knowledge runs a high risk of perpetrating a number of misinterpretations, logical blunders and impermissible comparisons that individuals knowledgeable in these fields would never make. However we are left with being curious how scale and probably other unknown factors contribute to the "logic" displayed by many organic and inorganic processes [16a] and why stochasticity need not interfere and indeed can contribute to the unfolding or probably better, emergence of affected cells? What is the relation, if any between scale and fractalgeometries associated with chaos [16b]? Are there any general principles that account for interactions between random, chance biologic events and their ability to find "interaction partners" that together mutually support these events and may advance the survival of the affected cell(s)? Do micro and macro- biologic and non-biologic events share these properties? We certainly don't know; perhaps somebody does.

\section{Acknowledgments}

We thank the Seidel Family Trust and Dr. Jules Harris for their support. Thanks are due to Dr. George Dunea, Director of the HektoenInstitute and Dr. Marvin Rubenstein Director of Cell Biology at the Hektoenwhere related work was performed. We thank Dr. Peter Hart, Director of the Renal Division and his staff at the Cook County Hospital, Chicago, Illinois for their support. We also thank Dr. Kevin Granfield of the University of Illinois College of Medicine, Chicago, Illinois for his editorial assistance. 


\section{References}

1. Davies, PCW (2004) Emergent biological principles and the computational properties ofthe universe. Complexity: 10, $11-15$.

2. Marshall I, Zohar D1 (997) Emergence, in Who's afraid of Schrodinger's cat? Quill, W Morrow, N Y. pgs 137139.

3. "Emergence". Wikipedia: The Free Encyclopedia. Wikimedia Foundation, Inc. Retrieved Summer, 2018.

4. op. cit. reference 2 Causation, 81-83.

5. Dozier, R. W (1992) Codes of Randomness.Crown Pub, N.Y.

6. Weinberg RA (2007) The biology and genetics of cells and organisms, chapter 1, 1-24 inThe Biology of Cancer, Garland Science, N Y.

7. Friedman- Morvinski, D, Bushong E A, Ke E et al. (2012) Dedifferentiation of neurons and astrocytes by oncogenes can induce gliomas in mice. Science 338:1080-1084.

8 a, b. (a) Eldredge N. The Pattern of Evolution (2018) Chapter 5, Of genes and species: Modern evolutionary theory. (b) "stochastic optimization" and "punctuated equilibria", ibid ref 3,Wikipedia, The Free Encyclopedia. Wikipedia Foundation, Inc . Retrieved Summer, 2018.

9. Schwartz, RS, Erban J K (2017) Timing of metastases in breast cancer.N E J M 376: 2486- 2488.

10. Laplane L (2014) Identifying some theories in developmental biology: the case of the cancer stem cell theory, 246-259 in Towards a Theory of Development, eds A Minelli, T Pradeau, Oxford Press.

11. Kupiec J-J (2014) Cell differentiation is a stochastic processsubject to natural selection, Ibid. 10:155-173.

12. a, b.(a) Luzzatto, I, Pandolfi P P (2015) Causality and chance in the development of cancer. N Eng J Med 373: 84-88.

(b) Nowak, M A, Waclaw B (2017) Genes, environment and "bad luck". Science 335:1266-1267.

13. YachidaS, Jones S, Bozic I et al. (2010) Distant metastases occurs late during thegenetic evolution of pancreatic cancer. Nature 467: 1114-1117.

14 a,b, c (a) Shain A H, Yeh I, Kovalyshyn L et al.(2015) The genetic evolution of melanoma from precursor lesions. $\mathrm{N}$ E J M 373: 1926-1936. (b) Gerlinger M, Rowan A J, Horswell $S$ et al. (2012) Intra-tumoral heterogeneity and branched chain evolution revealed in multi-region sequencing. $\mathrm{N} \mathrm{E} \mathrm{J}$ M 366: 883-892. (c) Tirosh I, Izar B, Prakadon S et al. (2016). Dissecting the multicellular ecosystem of metastatic melanoma by single-cell RNA- seq. Science 352: 189-196.
15. Nicolis G, Prigogine I (1989) Exploring Complexity. W H Freeman, N.Y. R Piper GmbH \& Co, KG Verlag, Munich.

16. a, b c. (a) West G (2018) Scale: The Universal Laws of Life, Growth and Death in Organisms, Cities and Companies. Penguin, N Y, N Y. 479 pp. (b) Chaos and selforganization, ibid ref 2, pgs. 83-85. c. Nikolic B K (2018) Phys. 460/660. Introduction to deterministic chaos. Dept. of physics and astronomy, University of Delaware.

17. Newman M E J (2010) Networks. Chapts 1,4,5,8. Oxford U. Press.

18. De vita V T, Lawrence T S, Rosenberg S A (2011) Cancer, Principles and Practice ofOncology in Primer of the Molecular Biology of Cancer. W Kluwer / Lippincott, William and Wilkins, Philadelphia, PA.

19. Hand D J (2014) The Improbability Principal, Chapter 3, What is chance?.41-74.Scientific American, Farrar, Strauss and Giroux, N Y, 41-74.

20. Anderson KM, Rubenstein M, Guinan P, Patel MK (2015) Contributions of stochasticevents to biologic evolution and cancer. J Can Res and Therapy 3, 105-111.

21. Coveney P, Highfield R (1990) Dissipative chaos, deterministic chaos The Arrow of Time. Fawcett Columbine,N Y.

22. Havlin S, Buldyev S V, Bunde A et al. (1999) Scaling in nature: from DNA through heartbeats to weather. Physica A 273, 36-69.

23. a, b. a. Mandelbrot B B(1983) The Fractal Geometry of Nature. W H Freeman, N Y. b Schroeder M(1910) Fractals, Chaos and Power Laws. Freeman, N Y.

24. Gleick J (1987) Chaos. Making of a New Science. Viking N Y.

25. "Chaos versus randomness", b. "chaos theory", "stochastic chaos", "quantum Bayesian chaos". ibid ref. 3. Wikipedia,accessed Summer, 2018.

26. Eldar A, Elowitz M B (2010) Functional role of noise in genetic circuits. Narture 467:167-173.

27. Sachez A, Golding I (2013) Genetic determinants and cellular constraints in noiseygene circuits. Science 342:11881193.

28. Gordon D J in DiVita V T, Lawrence, $\mathrm{T} S$, Rosenberg S A (2011) Primer of the Molecular Biology of Cancer. Mechanisms of genomic instability. Wolters Kluwer/ Lippincott, Williams \& Wilkens. Philadelphia, PA.

29. a - d (a) Tomasetti C. Vogelstein B (2015) Cancer etiology. Variations in cancer risk amngTissues can Be explained by the number of stem cell divisions. Science 347:78-80. (b) Tomasetti C, Li L., Vogelstein B (2017) Stem 
cell divisions, somatic mutations, cancer etiology, and cancer prevention. Science 355: 1330-1334. (c) Ayres R U (1994) Information, Entropy and Progress: A new Evolutionary Paradigm. Springer Verlag, Berlin. (d) Poach H A, Dellago C, Hoover W G, Kum O (1997) Microscopic time- reversal and macroscopic Irreversibility - Still a paradox? In Fleischhacker W, Schondeld T. (eds) Pioneering Ideas for the Physical and Chemical sciences, Springer, Boston MA.

30 Feinberg A P (2018) The key role of epigenetics in human disease prevention and mitigation. N E M J 378:13231334.

31. a, b,c, d Sell, S (1993) Cellular origin of cancer: dedifferentiation or stem cell maturation arrest? Environ Health Perspect 101 Suppl 5:15-26. (b) Martincorena I, Campbell P J. (2015) Somatic mutation in cancer and normal cells. Science 349:1483-1488. (c) Frank SA, Nowak M A (2004) Problems of somatic mutation and cancer. BioEssays 26: 291-299. (d) Freed D, Stevens E L, Pevsner J (2014) Somaticmosaicism in the human genome. Genes (Basel) 5: 1064-1094.

32. a, b (a) Gilbert S F (2006) Developmental Biology, $8^{\text {th }}$ ed. Sinauer Assoc., Sunderland, MA. (b) Lieberman B S, Eldredge N (2008) Punctuated Equilibria, Scholarpedia 3: 3806-3814.

33. Davidson E H. 2006 The Regulatory Genome. Acad. Press, N Y., Elsevier, Oxford

34. a, b (a) Kupper C, Stocks M, Russe J E et al. (2016) A supergene determines highly divergent male reproductive morphs in the ruff. Nature Gen 48, 79 - 83. (b) Pennisi E (2017) "Supergenes" drive evolution. Science 357,1083-1084.

35. a , b (a) Bennet K (2010) The chaos theory of evolution. New Scientist 13 Oct. (b) Rice S H, Papadopoulos A, Hartung J (2011) Stochastic processes driving directional evolution in Evolutionary Biology - Concepts, Biodiversity, Macroevolution and Genome Evolution, Springer - Verlag, Berlin and Heidelberg.

36. Pearse A, Swift K (2006) Transmission of devil-facialtumour disease. Nature 439: 549-553.

37. Rajasekhar V K, Vemuri M C, eds. (2009) Regulatory Networks in Stem Cells. Humana Press, Springer Science, NY.

38. Lanza R. (2009) (ed). Essentials of Stem Cell Biology, 2 ed. Elsevier A P, N Y, 2009.

39. Bridgeman, P W (1959) Some aspects of the physical sciences Chapt 5, 128-199, in The Way Things Are, Harvard U. Pres.

40. a, b Elowitz M B, Levine AJ, Siggia E D, Swain, P $S$ (2002) Stochastic gene expression in a single cell. Science 297:1183-1186. (b) Zong C, Lu S, Chapman A R, Xie, X S (2012) Genome-wide detection of single-nucleotide and copy-number variations of single human cell. Science 338: 1622-1626.

41. Lescroart F, Wang X, Lin, X et al. (2018) Defining the earliest steps of cardiovascularlineage segregation by single- cell RNA-seq. Science 359:1177-1181.

42. Robert L, Ollion J K, Robert J et al. (2018) Maturation dynamics and fitness effects followedIn single cells. Science 359: 1283-1286.

43. Fibib M G, Tirosh I, Hovestadt V et al. (2018) Developmental and oncogenic programs in H3K27M gliomas dissected by single - cell RNA-seq. Science 360: 331-335.

44. a-c (a) Fraga M F, Ballestar E, Paz M F et al. (2005) Epigenetic differences arise during thelifetimes of Monozygotic twins. Proc Nat Acad Sci 102: 10604-10609. (b) Zwijnenberg R J, Meijers-Heijboor H, Boomsma P I (2010) Identical but not the same: The value of discordantmonozygotic twins in genetic research. Am J. Med. Gen. 153 b, 1134-1149. (c) Chen Y C, SudreG, Sharp W et al (2015) Neuroanatomic, epigenetic and genetic differences in monozygotic Twins discordant for attention deficit hyperactivity syndrome. Mol Psych 23: 683690.

45. Casselman A (2008) Identical twins are not identical. Sci Am, April 3.

46. a, b (a) Foulkes W D, Real FX (2013) Many mosaic mutations. Current Oncology 20: 85-87(b) Abyzov A, Marian J, Palejev D et al. (2012) Somatic copy number mosaicism in human skin revealed by induced pluripotent stem cells. Nature 492: 438-442.

47. Everson T, Cole W (1968) Spontaneous Regression of Cancers. W B Saunders, Philadelphia PA.

48. Piel F B, Steinberg M H, Rees D C (2017) Sickle cell disease. N E J M 376, 1561-1573.

49. Baum, M (2015) Why does the weeping willow weep? Reconceptualizing oncogenesis in breast cancer. N E J M. 373: 1267-1269.

50. Davies, P (2008) The Goldilocks Enigma. Mariner Book. Houghton Mifflin Co., Boston, N.Y.

51. a, b. (a). Park J W, Lee J K, Shen K M et al. (2018) Reprogramming normal human epithelial tissues to a common lethal neuroendocrine cancer lineage. Science 362: 91-95. (b) Kareta R S, Sage J. ibid 51, pgs 30-31. 
Submit your manuscript to a JScholar journal and benefit from:

ฯ Convenient online submission

- Rigorous peer review

- Immediate publication on acceptance

- Open access: articles freely available online

ฯ High visibility within the field

ब Better discount for your subsequent articles Submit your manuscript at http://www.jscholaronline.org/submit-manuscript.php 